\title{
Design of U-PPC-Type II for Nonlinear Systems
}

\author{
ZHU Ge ${ }^{1}$, QIU Ji², ZHU Quanmin ${ }^{3,}$, NARAYAN Pritesh ${ }^{4}$, WRIGHT Steve ${ }^{5}$, \\ 1. Department of Engineering Design and Mathematics, University of the West of England, Frenchay Campus, Coldharbour Lane, Bristol, \\ BS16 1QY, UK \\ E-mail: Ge2.Zhu@uwe.ac.uk, Ji2.Qiu@uwe.ac.uk, Quan.Zhu@uwe.ac.uk, Pritesh.Narayan@uwe.ac.uk, Steve.Wright@uwe.ac.uk,
}

\begin{abstract}
In this study, a new U-PPC-Type II (U-model Pole Placement Control Type II) control system design procedure is proposed based on the U-model principle. The objective of a U-PPC-Type II design is to determine a linear controller $G_{c}$ from a specified closed loop linear transfer function $G_{c l s}$. The study also compares the new design procedure with a U-PPC-Type I based design procedure. For demonstration of the effectiveness of the proposed new procedure, U-PPC-Type II is designed for both a linear dynamic model and a Hammerstein (nonlinear dynamic) model. The simulation results are presented with discussions and graphical illustrations.
\end{abstract}

Key Words: U-model, Pole placement, U-PPC-Type I, U-PPC-Type II, Nonlinear dynamic control, Linear Dynamic control

\section{Introduction}

The Control of nonlinear dynamic systems has long been recognised as a challenging issue. The key design objective in nonlinear control systems is a general model prototype with conciseness, flexibility and manipulability, while maintaining little loss of precision. This was the original motivation for proposing the U-model in one of the authors' $\mathrm{PhD}$ thesis [1] and the first journal paper that appeared in 2002 [2], with a U-Model and pole placement controller design for nonlinear dynamic plants [2]. The research progression over a decade is illustrated by two recent review publications [3, 4]. The U-model covers almost all existing smooth nonlinear discrete time polynomial models as its subsets. Consequently, linear polynomial model-based design approaches can be directly used to design such nonlinear control systems $[5,6]$.

Motivated by some previous theoretical results $[1,2,5$, 6], a computational complexity in designing pole placement controller with U-model has been observed that, even once off, a Diophantine equation still needs to be resolved. This study is devoted to designing, still within a U-model framework, a pole placement controller, but without resolving a Diophantine equation. Furthermore, the new controller enables more efficient construction of closed loop control by using a classical error signal (that is, the difference between reference and output). To justify the study explained above, a few of the original research questions are listed, which subsequently guided the study to provide possible solutions.

Research Question One: How can a pole placement control be designed without using a Diophantine equation?

Research Question Two: How can a conventional error signal (that is, the difference between reference and plant output) be effectively used against that used in classical pole placement control design [9]?

${ }^{*}$ Corresponding author

This work is partially supported by the National Nature Science Foundation of China under Grant 61273188 and Taishan Scholar Funding, Shandong, China.
Research Question Three: Are there some bench tests available to demonstrate the effectiveness and efficiency of U-PPC-Type II?

The main contents of this paper are divided into three sections. In section 2 an existing control-oriented U-model is briefly outlined, representing a wide range of nonlinear plants. This is briefly outlined to lay a foundation for further development. In section 3 a general U-PPC-Type II design procedure based the U-model framework is designed. In section 4 a linear dynamic plant and a nonlinear dynamic Hammerstein model is selected to demonstrate the design procedure.

\section{U-Model}

\subsection{Description of U-Model}

Consider a general single-input and single-output nonlinear dynamic polynomial plant described as follows:

$$
\begin{aligned}
& y(t)=f(P(*), \Theta) \\
& P(*)=P(y(t-1), \ldots, y(t-n), u(t-1), \ldots, u(t-n))
\end{aligned}
$$

Where $y(t) \in R$ and $u(t-1) \in R$ are the output and input signals of the plant respectively (also known as the controller output in the control system design), at discrete time instant $t(1,2, \ldots), n$ is the plant order. $P(*)=P(y(t-1), \ldots, y(t-n), u(t-1), \ldots, u(t-n)) \in R^{L+1}$ is the regression variable vector spanned from the delayed outputs and inputs and $\Theta=\left[\theta_{0} \ldots \theta_{L}\right] \in R^{L+1}$ is the associated parameter vector. The model can be further expressed in terms of a regression equation as:

$$
y(t)=\sum_{l=0}^{L} p_{l}(t) \theta_{l}
$$

Where the regression terms $p_{l}(t)$ are the products of past inputs and outputs, such that $u(t-1) y(t-3), u(t-1) u(t-2), y^{2}(t-1)$ and $\theta_{l}$ are the associated parameters. It is should be noted that this 
model set has been widely accepted as NARMAX (nonlinear auto-regressive moving average) models [7].

The U-model is defined as, under a $U$ mapping from the above polynomial model, a controller output $u(t-1)$ oriented polynomial below:

$$
\begin{aligned}
& y(t)=f(\mathrm{Y}(*), U(t-1)) \\
& \mathrm{Y}(*)=\mathrm{Y}(\mathrm{y}(\mathrm{t}-1), \ldots, \mathrm{y}(\mathrm{t}-\mathrm{n}), \mathrm{u}(\mathrm{t}-2), \ldots, \mathrm{u}(\mathrm{t}-\mathrm{n}), \Theta) \\
& U(t-1)=\left(\operatorname{constu}(t-1) u^{2}(t-1) \ldots u^{M}(t-1)\right.
\end{aligned}
$$

In order to apply linear control system design methodology to a nonlinear model, there is [1]:

$$
y(t)=U(t)
$$

The control oriented NARMA expression is proposed by expanding the nonlinear function $f($.$) as a polynomial with$ respect to $u(t-1)$ as follows:

$$
y(t)=\sum_{j=0}^{M} \lambda_{j}(t) u^{j}(t-1)
$$

Where $M$ is the degree of model input (controller output) $u(t-1)$, the time varying parameter vector $\lambda(t)=\left[\lambda_{0}(t) \ldots \lambda_{M}(t)\right] \in R^{M+1}$ is a function of past inputs and outputs $(u(t-2), \ldots, u(t-n), y(t-1), \ldots, y(t-n)) \quad$ and the parameters $\left(\theta_{0} \ldots \theta_{L}\right)$ in (2.2). Such an example, from the polynomial model to the U-model conversion, is shown below.

The polynomial model is [3]

$$
\begin{aligned}
y(t)= & 0.1 y(t-1) y(t-2)-0.5 y(t-1) u^{2}(t-1) \\
& +0.8 u(t-1) u(t-2)
\end{aligned}
$$

And the U-model can be determined in the notation of (2.5) [5],

$$
y(t)=\lambda_{0}(t)+\lambda_{1}(t) u(t-1)+\lambda_{2}(t) u^{2}(t-1)(2.7)
$$

Where $\lambda_{0}(t)=0.1 y(t-1) y(t-2), \lambda_{1}(t)=0.8 u(t-2)$ , and $\lambda_{2}(t)=-0.5 y(t-1)$.

\subsection{U-Control System Design Procedure}

In order to use linear polynomial model-based design approaches, the desired plant output is defined as $y_{d}(t)$, which is specified either by designers or customers in advance. Accordingly, the relationship between a specified plant output $y_{d}(t)$ and the requested corresponding controller output $u(t-1)$ can be expressed in terms of the U-model,

$$
y_{d}(t)=\sum_{j=0}^{M} \lambda_{j}(t) u^{j}(t-1)
$$

Accordingly, this establishes a prototype for proposing a new two-step design procedure [6].

Step 1 (Design $y_{d}(t)$ ): The first task of the design is to determine the desired plant output $y_{d}(t)$ according to a specified performance index (for example, Pole Placement Control (PPC) [2]). The PPC has been designed in terms of

$$
R y_{d}(t)=T w(t)-S y(t)
$$

Where $y(t), y_{d}(t)$ and $w(t)$ are the plant output, desired/designed plant output and reference input respectively. The polynomials, $\mathrm{R}, \mathrm{S}$ and $\mathrm{T}$ are used to specify the desired plant output $y_{d}(t)$

Step 2 (Work out $u(t-1)$ ): Then, the task is to resolve one of the roots of the formula (2.8) to obtain the controller output $u(t-1)$ [6].

$$
u(t-1)=\Psi\left[y_{d}(t)-\sum_{j=0}^{M} \lambda_{j}(t) u^{j}(t-1)=0\right]
$$

Where $\Psi[*]$ is a root-solving algorithm, such as Newton-Raphson algorithm [8]. A detailed analysis on the root solving issues has been presented [2].

Block diagram Figure 1 shows a general U-model based pole placement control system structure with the proposed design procedure.

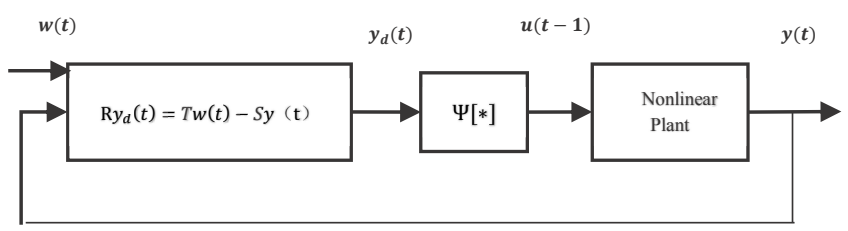

Figure 1 U-model based pole placement control system.

\section{Design of U-PPC-Type II}

\subsection{U-PPC-Type I}

A U-PPC-Type I model is converted from nonlinear polynomial model through a U-model expression (2.5) and assigned with required poles through a linear feedback control algorithm. By letting $\mathrm{y}(t)=y_{d}(t)$, the function of the U-PPC-Type I model as show as below:

$$
\begin{aligned}
& \mathrm{y}(\mathrm{t})=y_{d}(t)=\frac{T}{R+S} w(t)=\frac{T}{A_{c}} w(t) \\
& A R+B S=A_{c}
\end{aligned}
$$

where $w(t)$ is the reference input, $y(t)$ is system output, $y_{d}(t)$ is the desired plant output and $\mathrm{R}, \mathrm{S}$ and $\mathrm{T}$ are the polynomial of the forward shift operator. The polynomial $A_{c}$ is the closed loop characteristic equation. The structure of U-PPC-Type I was shown as Figure 1.

To cancel the possible output o set in steady state, i.e., to make the steady state error equal to zero at the controlled output, polynomial $\mathrm{T}$ is specified with

$$
T=A_{c}(1)
$$

The key idea of the U-PPC-Type I model design is to specify the desired closed-loop characteristic polynomial $A_{c}$ , then resolve the polynomials $\mathrm{R}$ and $\mathrm{S}$ through a Diophantine equation [2]. After designing the desired plant output $y_{d}(t)$, the controller output $u(t-1)$ can be determined by resolving one of the roots of the U-model expression (2.8). 
Theorem: The U-model based pole placement design procedure does not depend on the plant model. Only the solution of the designed controller output involves the plant model [6].

Proof: This directly comes from the relationship of (3.1). That is easy to deduce the relationship as this equation:

$$
R+S=A_{c}
$$

In a classical approach [9], the corresponding relationship is given by

$$
A R+B S=A_{c}
$$

where $A$ and $B$ are the numerator polynomial and the denominator polynomial of a plant model, respectively, which indicate the classical design depending on the plant model.

Actually Theorem provides a new design framework, using the U-control design procedure once, then it can be applied to many different plant models, which only calculates the corresponding controller output each time from a given plant model [6].

\subsection{U-PPC-Type II}

As mentioned in the previous section, the conception of the U-model Pole Placement Control Type II (U-PPC-Type II) is based on understanding the properties of the U-PPC-Type I model, such as the specifically desired closed-loop characteristic polynomial, and work out the desired plant output $y_{d}(t)$ and thus the controller output $u(t-1)$.

Generally speaking, the U-PPC-Type II is the transformation and simplification of the U-PPC-Type I model, that is designing the desired plant output $y_{d}(t)$ without resolving the Diophantine equation, and directly determined desired transfer function, by using a linear feedback control algorithm.

In the U-PPC-Type II design procedure, after specification of the desired closed-loop characteristic polynomial $A_{c}$, and by letting $\mathrm{y}(t)=y_{d}(t)$, according to reference expressions (3.1) and (3.2) the assigned numerator polynomial of the U-PPC-Type II is:

$$
T=A_{c}(1)
$$

Correspondingly, the U-PPC-Type II desired closed-loop transfer function $G_{c l s}$ is

$$
G_{c l s}=\frac{y(t)}{w(t)}=\frac{T}{A_{c}}=\frac{A_{c}(1)}{z^{k}+a_{c 1} z^{k-1}+\ldots a_{c k}}
$$

Where $\left[a_{c 1} \ldots a_{c k}\right]$ are coefficients of polynomial $A_{c}$.

In this study the desired open-loop transfer function $G_{c}$ can be derived by $G_{c l s}$, the feedback relational expression as below:

$$
G_{c l s}=\frac{G_{c}}{1+G_{c}}
$$

The Figure 2 shows the basic relationship between open-loop transfer function $G_{c}$ and desired closed-loop transfer function $G_{c l s}$ in the ideal condition, by letting $\mathrm{y}(t)=y_{d}(t)$ :

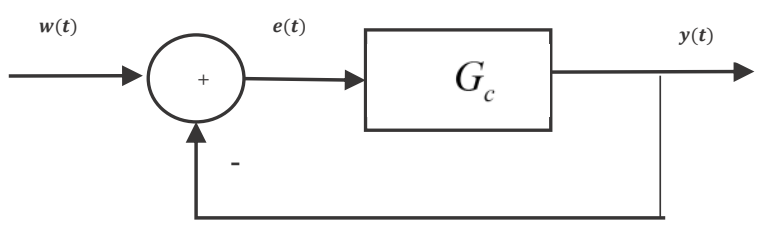

Figure 2 Close-loop system based open-loop transfer function $G_{c}$

According to expression (3.6) and (3.7), the derived desired open-loop transfer function is:

$$
G_{c}=\frac{G_{c l s}}{1-G_{c l s}}=\frac{A_{c}(1)}{z^{k}+a_{c 1} z^{k-1}+\ldots a_{c k}-A_{c}(1)}
$$

Block diagram Figure 3 shows a general U-PPC-Type II based U-model control system structure with the proposed design procedure.

Where $e(t)$ is the actuating error signal, the relational expression with reference input $w(t)$ and $y(t)$ is:

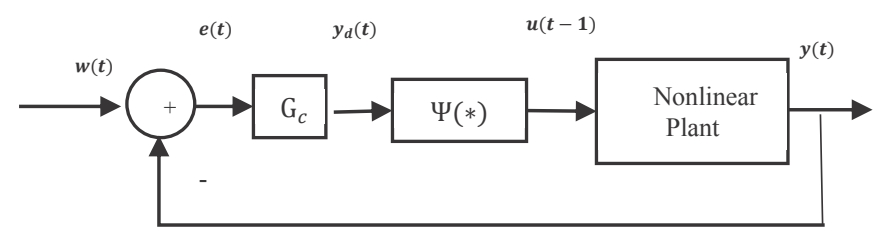

Figure 3 U-PPT-Type II based U-model control system.

$$
e(t)=w(t)-y(t)
$$

From Figure 3, the relationship between $e(t), y_{d}(t)$ and desired open-loop transfer function $G_{c}$ can be deduced:

$$
G_{c}=\frac{y_{d}(t)}{e(t)}
$$

The key idea of the U-PPC-Type II is to specify the desired closed-loop characteristic polynomial $A_{c}$ and substitute it into the desired closed-loop transfer function $G_{c l s}$ : then the desired open-loop transfer function $G_{c}$ can be derived from $G_{c l s}$. After derived $G_{c}$, one could work out the desired plant output $y_{d}(t)$. The controller output $u(t-1)$ can be determined by resolving one of the roots of the U-model expression (2.8).

\subsection{Comparison of U-PPC-Type-I and U-PPC-Type II}

With reference to U-PPC-Type I and U-PPC-Type II, as presented with section 3.1 and section 3.2 , there is a very clear picture distinguishing the two procedures from their structures. For U-PPC-Type I, the key point of the design is to specify the desired closed-loop characteristic polynomial 
$A_{c}$ : the polynomial $\mathrm{R}$ and $\mathrm{S}$ can then be resolved through a Diophantine equation. The difficulty is that the equation is linear in the polynomial of $\mathrm{R}$ and $\mathrm{S}$. A solution to the equation exists. However, the equation has many other solutions [9]. Therefore designers must select a specific solution by imposing some constraints on these general solutions. Secondly, the error signal is not in a convenient format for industrial applications, as generation of the particular error signal requires the assignment of two polynomials ( $R$ and $S$ ), which further increases the complexity of the controller design.

U-PPC-Type II has presented an intuitive linear feedback control design strategy and directly reflected on its conception design. U-PPC-Type II can avoid those constrains imposed by U-PPC-Type I. Therefore it specifies the desired close-loop characteristic polynomial $A_{c}$ and directly deduces the desired open-loop transfer function $G_{c}$ from $G_{c l s}$, and consequently the desired plant output $y_{d}(t)$ and the controller output $u(t-1)$.

\section{Case Studies - Design and Simulation}

A linear dynamic plant and a Hammerstein model were selected to test the design methodology. The same closed-loop specifications were assigned for these models, to demonstrate that the proposed method is generally suitable for controlling different dynamics.

In theory, the U-model-based design procedure does not require the plant model (whether linear or nonlinear). The specifically desired closed-loop characteristic equation is:

$$
A_{c}=z^{2}-1.3205 z+0.4966
$$

Therefore, the closed-loop poles are a complex conjugate pair of $-0.6603 \pm j 0.2463$. This design specification corresponds to a natural frequency of $1 \mathrm{rad} / \mathrm{sec}$ and a damping ratio of 0.7 . To achieve zero steady state error, specify:

$$
T=A_{c}(1)=1-1.3205+0.4966=0.1761
$$

Therefore, the desired closed-loop transfer function is:

$$
G_{c l s}=\frac{0.1761}{z^{2}-1.3205 z+0.4966}
$$

According to expression (3.6):

$$
G_{c}=\frac{G_{c l s}}{1-G_{c l s}}=\frac{0.1761}{z^{2}-1.3205 z+0.3205}
$$

Substituting the coefficients of the transfer function $G_{c}$ into the controller of (2.7) give rise to:

$$
\begin{aligned}
y_{d}(t+1)= & 1.3205 y_{d}(t)-0.3205 y_{d}(t-1) \\
& +0.1761 e(t-1)
\end{aligned}
$$

The controller output $u(t)$ can be determined from resolving (2.9).

\subsection{Control of the Linear Dynamic Model}

Consider a linear dynamic plant described by the following model,

$$
y(t)=1.2 y(t-1)-0.4 y(t-2)+u(t-1)+0.5 u(t-2)
$$

The corresponding control oriented model is obtained from relational expression (2.4), it gives:

$$
y(t)=\lambda_{0}(t)+\lambda_{1}(t) u(t-1)
$$

Where

$$
\begin{aligned}
& \lambda_{0}(t)=1.2 y(t-1)-0.4 y(t-2)+0.5 u(t-2) \\
& \lambda_{1}(t)=1
\end{aligned}
$$

For linear plant model [6], it has, from expression (2.7),

$$
u(t-1)=\frac{y_{d}(t)-\lambda_{0}(t)}{\lambda_{1}(t)}
$$

In this simulation, a periodic square wave was selected to be the reference signal, to be followed by the system output. The simulation results are shown in Figures 4 and 5, where Figure 4 gives the system response and Figure 5 the controller output. The simulation results show that the proposed control strategy provides a stable system performance.

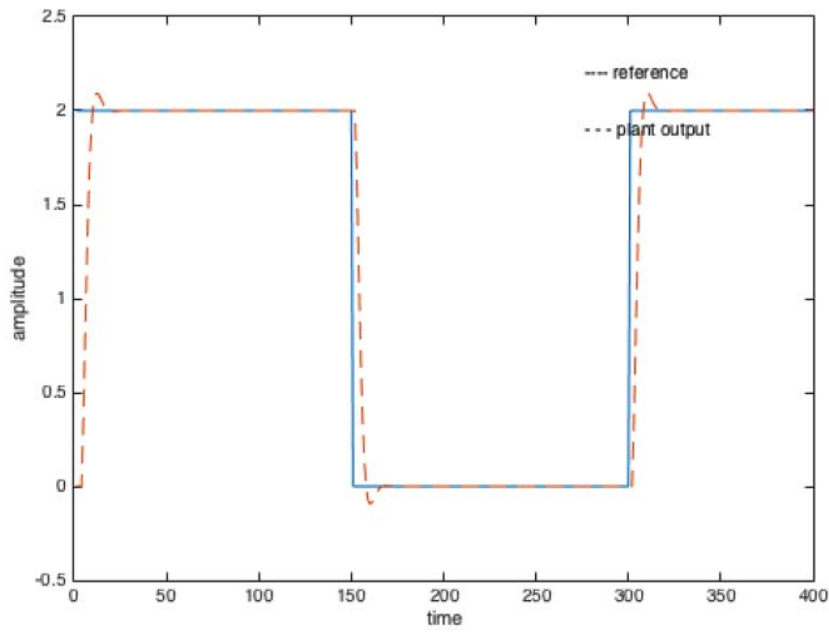

Figure 4 System output of the linear dynamic control system.

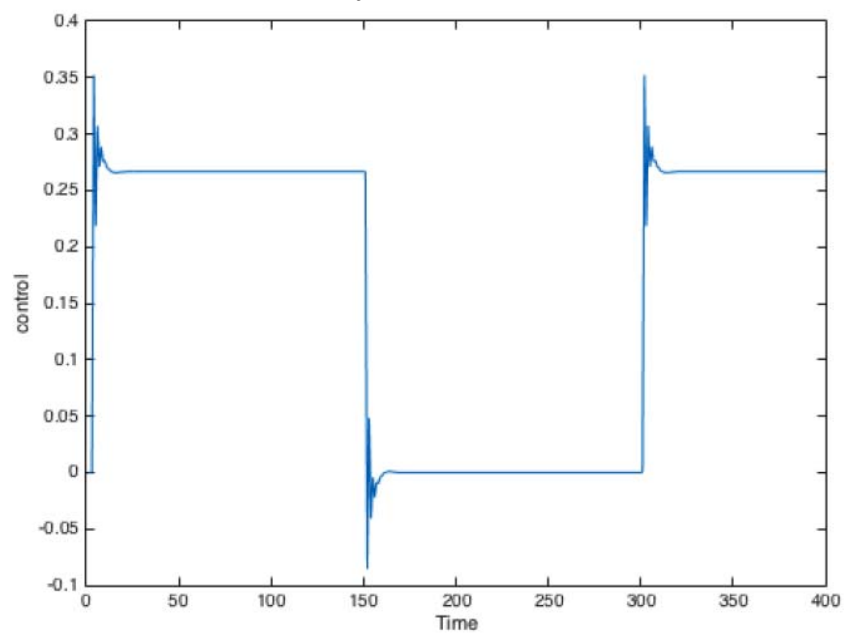

Figure 5 Controller output of the linear dynamic control system.

\subsection{Control of the Hammerstein Model}

Consider a nonlinear dynamic plant described by the following Hammerstein model, 


$$
\begin{aligned}
& y(t)=0.5 y(t-1)+x(t-1)+0.1 x(t-2) \\
& x(t)=1+u(t)-u^{2}(t)+0.2 u^{3}(t)
\end{aligned}
$$

The first step is to covert the Hammerstein model into the U-model, with reference expression (2.4)

$$
\begin{aligned}
y(t)=\lambda_{0}(t) & +\lambda_{1}(t) u(t-1)+\lambda_{2}(t) u^{2}(t-1) \\
& +\lambda_{3}(t) u^{3}(t-1)
\end{aligned}
$$

Where

$$
\begin{aligned}
& \lambda_{0}(t)=0.5 y(t-1)+1+0.3 x(t-2) ; \lambda_{1}(t)=1 ; \\
& \lambda_{2}(t)=-1 ; \lambda_{3}(t)=0.2
\end{aligned}
$$

The controller output $u(t)$ is determined by solving

$$
y_{d}(t+1)-\lambda_{0}(t+1)-u(t)+u^{2}(t)-0.2 u^{3}(t)=0
$$

The derivative of $y_{d}(t+1)$ against $u(t)$ is required in the Newton-Raphson root solving algorithm [5], which is given by:

$$
\frac{d\left[y_{d}(t+1)\right]}{d u(t)}=3 \lambda_{3}(t+1) u^{2}(t)-2 u(t)+1
$$

Figure 6 gives the system response under the proposed U-PPC-Type II control. It can be seen from the simulation result that the resultant closed-loop system behaves similarly to that of a linear system. This is due to cancellation of the nonlinearity by the proposed control oriented model and controller design approach.

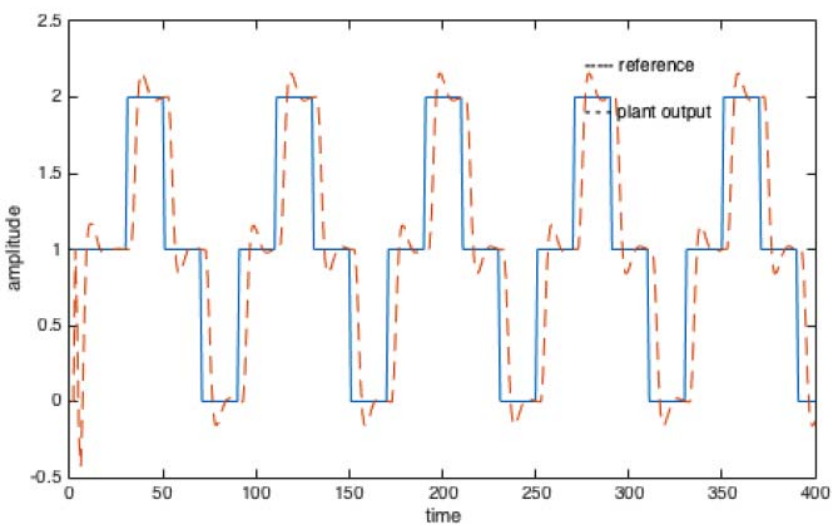

Figure 6 System response of the Hammerstein model.

\section{Conclusion}

A general U-PPC-Type II design procedure has been proposed to simplify and generalise the U-PPC-Type I design procedure. U-PPC-Type II transforms U-model pole placement into a classical closed-loop control. U-PPC-Type
II has several favourable properties, such as elimination of the resolving of Diophantine equations, and using classical error signals to form closed loop control systems. This gives clear insight and understanding into U-model based control system design and classical closed loop implementation. Finally, the U-PPC-Type II enhanced U-model control system makes U-control more flexible, manipulative and concise in control of linear and nonlinear dynamic systems.

\section{Acknowledgements}

The first two authors are grateful to the partial studentship sponsored by the Engineering Modelling and Simulation Research Group, University of the West of England, Bristol, UK. The third author is grateful to the support by the National Nature Science Foundation of China under Grant 61273188 and Taishan Scholar Construction Engineering Special Funding, Shandong, China.

\section{References}

[1] Zhu, Q.M., Identification and Control of nonlinear systems, $\mathrm{PhD}$ thesis, University of Warwick, UK, 1989.

[2] Zhu, Q.M. and Guo, L.Z., A pole placement controller for nonlinear dynamic plants, Journal of Systems and Control Engineering, Proceedings of the Institution of Mechanical Engineers Part I, 216(6): 467-476, 2002.

[3] Xu, F.X., Zhu, Q.M., Zhao, D.Y., and Li, S.Y., U-model based design methods for nonlinear control systems-A survey of the development in the 1st decade, Control and Decision, Vol. 28, No. 7, 961-971, 2013 (in Chinese).

[4] Zhu, Q.M., Wang, Y.J,, Zhao, D.Y., Li, S.Y., and Billings, S.A., Review of rational (total) nonlinear dynamic system modelling, identification and control, Int. J. of Systems Science, 2015 (in press).

[5] Du, W.X., Wu, X.L, and Zhu, Q.M., Direct design of $U$-model based generalized predictive controller (UMGPC) for a class of nonlinear (polynomial) dynamic plants, Proc. Instn. Mech. Enger, Part I: Journal of Systems and Control Engineering, 226: 27-42, 2012.

[6] Zhu, Q.M., Zhao, D.Y., and Zhang, J.H., A general U-block model-based design procedure of nonlinear polynomial control systems, International Journal of Systems Science, 10.1080/00207721.2015.1086930, 2015.

[7] Billings, S.A., Nonlinear System Identification: NARMAX Methods in the Time, Frequency, and Spatio-Temporal Domains, Wiley, John \& Sons, Chichester, West Sussex, 2013.

[8] Chong, E.K.P. and Zak, S.H., An Introduction to Optimization, (4th ed.), Wiley, Purdue University, 2013

[9] Astrom, K.J. and Wittenmark, B., Adaptive control (2nd ed). Reading: Dover, 1995. 